\title{
Gravitational Anomalies and the Family's Index Theorem
}

\author{
Orlando Alvarez ${ }^{*}{ }^{\star}$, I. M. Singer ${ }^{2}$, and Bruno Zumino ${ }^{1}$ \\ 1 Department of Physics and Lawrence Berkeley Laboratory, University of California, Berkeley, \\ CA 94720, USA \\ 2 Department of Mathematics and Department of Physics, University of California, Berkeley, \\ CA 94720, USA
}

\begin{abstract}
We discuss the use of the family's index theorem in the study of gravitational anomalies. The geometrical framework required to apply the family's index theorem is presented and the relation to gravitational anomalies is discussed. We show how physics necessitates the introduction of the notion of local cohomology which is distinct from the ordinary topological cohomology. The recent results of Alvarez-Gaumé and Witten are derived by using the family's index theorem.
\end{abstract}

\section{Introduction}

Alvarez-Gaumé and Witten [1] have calculated the gravitational anomalies of certain parity violating theories in $4 k-2$ dimensions. Their most striking result is that there is a unique minimal ten dimensionl theory where the gravitational anomalies cancel. In this communication we reproduce their results in a different way by using the family's index theorem [2] instead of Feynman diagram methods.

The relation of the family's index theorem to anomalies has been discussed by Atiyah and one of the present authors in reference [3]. In that paper, the geometric setting for the family's index theorem was presented and the relation to anomalies was discussed. The authors showed that the first characteristic class of the index bundle for the Dirac operator was related to anomalies. A number of papers have addressed the relationships among chiral anomalies, the geometry of the space of vector potentials, and the families of Dirac operators. We recommend the papers of Alvarez-Gaumé and Ginsparg [4], Lott [5], and Stora [6] to the reader. The first investigation of the behavior of the Dirac operator as a function of the metric is due to Hitchin [7].

* This work was supported in part by the National Science Foundation under Contracts PHY8118547 and MCS80-23356; and by the Director, Office of High Energy and Nuclear Physics of the US Department of Energy under Contracts DE-AC03-76SF00098 and AT0380-ER10617

$\star \star$ Alfred P. Sloan Foundation Fellow 
This is a brief report of a more extensive study we have done on the relation of the family's index theorem to anomalies. In preparation is an expository paper [8] which we hope will be accessible to physicists. In this note we assume some knowledge of K-theory and characteristic classes. Physicists may find a useful discussion of these topics in the review article by Eguchi, Gilkey, and Hanson [9].

\section{Families of Metrics and the Family's Index Theorem}

Let $M$ be a $2 n=4 k-2$ dimensional spin manifold. The space of allowable metrics on $M$ will be denoted by $\mathfrak{M}$. The diffeomorphism group of $M$ is denoted by $\operatorname{Diff}(M)$. For our purposes we require a subset of Diff $(M)$ denoted by Diff ${ }_{0}(M)$ and defined by

$$
\begin{aligned}
& \operatorname{Diff}_{0}(M) \\
& \quad=\left\{\phi \in \text { identity component of } \operatorname{Diff}(M): \phi\left(x_{0}\right)=x_{0}, d \phi\left(x_{0}\right)=i d\right\} .
\end{aligned}
$$

where $x_{0} \in M$ is a fixed reference point and $d \phi$ is the differential of $\phi$. Later we will explain the reason for working with $\operatorname{Diff}_{0}(M)$.

We will be interested in the properties of a two parameter family of elliptic operators on $M$. The operators in question are the chiral Dirac operators coupled to a vector bundle $V$ associated with the spin frame bundle. To be more precise, let $S_{M}^{ \pm}$ be the positive and negative chirality spin bundles over $M$. Let

$$
B_{g}: C^{\infty}\left(S_{M}^{+} \otimes V\right) \rightarrow C^{\infty}\left(S_{M}^{-} \otimes V\right)
$$

be the chiral Dirac operator coupled to $V$. The subscript $g$ is a reminder that the Dirac operator depends on the metric $g$. A two parameter family of operators will be generated by varying the metric. The action of $\phi$ on $g$ is denoted by $\phi \cdot g$. Since $B_{g}$ is covariant under the action of $\operatorname{Diff}(M)$,

$$
B_{\phi \cdot g}=\phi^{-1} B_{g} \phi,
$$

we will have to be careful in specifying the two parameter family.

The space of metrics $\mathfrak{M}$ is a principal fiber bundle over $\mathfrak{M} / \operatorname{Diff}_{0}(M)$ with structure group $\operatorname{Diff}_{0}(M)$. The reason for choosing $\operatorname{Diff}_{0}(M)$ is that $\operatorname{Diff}(M)$ does not act freely on $\mathfrak{M}$. In fact, if $\phi \in \operatorname{Diff}(M)$ is an isometry of $g$, then the action of $\phi$ on $g$ is by definition $\phi \cdot g=g$. The only isometry in $\operatorname{Diff}_{0}(M)$ is the identity transformation, and therefore $\operatorname{Diff}_{0}(M)$ acts freely on $\mathfrak{M}$. Diff $(M)$ could be used if we restricted the metrics to those with no isometries besides the identity.

The family of operators $B_{g}$ has a family of kernels, $\operatorname{ker} B_{g}$, the zero frequency modes of positive chirality. There is also the family ker $B_{g}^{\dagger}$, the zero frequency modes of negative chirality for $B_{g}^{\dagger}$. Because $B_{\phi \cdot g}=\phi^{-1} B_{g} \phi$, the vector space $\operatorname{ker} B_{\phi \cdot g}=$ $\phi\left(\operatorname{ker} B_{g}\right)$; we identify them so that one obtains two families of vector space ker $\mathbf{B}_{\rho}$ and $\operatorname{ker} \mathbf{B}_{\rho}^{\dagger}$ indexed by $\rho \in \mathfrak{M} / \operatorname{Diff}_{0}(M)$. Although these families can jump in dimension, their formal difference $\operatorname{ker} \mathbf{B}_{\rho}-\operatorname{ker} \mathbf{B}_{\rho}^{\dagger}$ makes sense in $\mathrm{K}$-theory, and is the index bundle, Ind $\left(\mathbf{B}_{\rho}\right)$.

The non-vanishing of the Chern classes of Ind $\left(\mathbf{B}_{\rho}\right)$ measures the extent $\operatorname{ker} \mathbf{B}_{\rho}$ and ker $\mathbf{B}_{\rho}^{\dagger}$ differ. The first Chern class is a two form on $\mathfrak{M} / \operatorname{Diff}_{0}(M)$ and has a simple physical interpretation. Suppose $\operatorname{ker} \mathbf{B}_{\rho}$ is of dimension $r$ with zero frequency modes 
$\psi_{1}, \ldots, \psi_{r}$. The exclusion principle requires one to study the antisymmetric combination $\psi_{1} \wedge \cdots \wedge \psi_{r}$, and hence the one dimensional (line) bundle $\Lambda^{r} \operatorname{ker} \mathbf{B}_{\rho}$ is important. Mathematically, if $\operatorname{ker} \mathbf{B}_{\rho}^{\dagger}$ has dimension $s$, then the line bundle $\Lambda^{r} \operatorname{ker} \mathbf{B}_{\rho} / \Lambda^{s} \operatorname{ker} \mathbf{B}_{\rho}^{\dagger}$ is well defined even when there is a jump (of the same dimension) in $\operatorname{ker} \mathbf{B}_{\rho}$ and $\operatorname{ker} \mathbf{B}_{\rho}^{\dagger}$ (the effects of the jumps cancel). This line bundle is called the determinant line bundle of $\mathbf{B}_{\rho}$ which we denote by DET Ind $\mathbf{B}_{\rho}$. We use the boldface DET in order not to confuse the above with the functional determinant of an operator. The first Chern class $c_{1}$ of Ind $\mathbf{B}_{\rho}$ is the first Chern class of DET Ind $\mathbf{B}_{\rho}$ and one can evaluate it by restricting to two dimensional spheres $Y$ in $\mathfrak{M} / \operatorname{Diff}_{0}(M)$.

What this boils down to is the following. Let $Y_{ \pm}$be the upper and lower hemispheres of $Y$ parametrizing metrics. Along the equator we assume the metric $\rho_{-}(\theta)$ from the lower hemisphere equals $\phi(\theta) \cdot \rho_{+}(\theta)$, where $\rho_{+}(\theta)$ is the metric from the upper hemisphere, and $\phi(\theta) \in \operatorname{Diff}_{0}(M)$. In the above discussion $\theta$ is the equatorial angle. Correspondingly, patch $\operatorname{ker} \mathbf{B}_{\rho}, \rho \in Y_{-}$along the equator by $\operatorname{ker} \mathbf{B}_{\rho_{-}(\theta)}=\phi \cdot \operatorname{ker} \mathbf{B}_{\rho_{+}(\theta)}$. Do the same for $\operatorname{ker} \mathbf{B}_{\rho}^{\dagger}$. In this manner we get two families of vector spaces $E$ and $F$ over $Y$ whose formal difference is the restriction of Ind $\mathbf{B}_{\rho}$ to $Y \subseteq \mathfrak{M} \operatorname{Diff}_{0}(M)$. In the same way, $\operatorname{DET}(E-F)$ is the restriction of DET Ind $\mathbf{B}_{\rho}$ to $Y$. Note that this construction leads automatically to the construction of a fibre bundle $Z$ with base $Y$ and fibre $M$. A trivialization of $Z$ is obtained by looking at $Y_{ \pm} \times M$. The bundle's transition function at the equator is given by $\phi(\theta)$. Note that $Z$ is $4 k$ dimensional. A corollary of the family's index theorem is that

$$
\left.\operatorname{ch}\left(\operatorname{Ind} \mathbf{B}_{\rho}\right)\right|_{Y}=\int_{M} \widehat{A}(Z) \operatorname{ch}(V) \text {. }
$$

$\operatorname{ch}\left(\operatorname{Ind} \mathbf{B}_{\rho}\right)$ is the Chern character for the index bundle. On the right-hand side of the equation one has the $\hat{A}$ genus for the manifold $Z$, and the Chern character of $V$ which should now be thought as a vector bundle over $Y$. One should interpret (2.4) in terms of differential forms. Since $Y$ is two dimensional one has that $\left.\operatorname{ch}\left(\operatorname{Ind} \mathbf{B}_{\rho}\right)\right|_{Y}=$ $v+c_{1}\left(\operatorname{Ind} \mathbf{B}_{\rho}\right)$, where $v$ is the ordinary index of each operator and is independent of $\rho$. From now on we can restrict ourselves to the family $\left\{\mathbf{B}_{\rho}\right\}$ for $\rho \in Y$.

\section{Relation of the Family's Index Theorem to the Anomaly}

The gravitational anomaly also called the Einstein anomaly is the variation of the $\log \operatorname{det} B_{g}$ under a diffeomorphism. If $\xi$ is the vector field on $M$ that generates a diffeomorphism, then the anomaly is given by

$$
\int_{M} d(\operatorname{vol}) \xi^{\mu}(x) \nabla^{v} T_{\mu v},
$$

where $T_{\mu \nu}$ is the energy momentum tensor. The anomaly is a one form on $\operatorname{Diff}_{0}(M)$, since (3.1) is the evaluation of a one form on a tangent vector to $\operatorname{Diff}_{0}(M)$. The consistency condition [10] generalized to the case of diffeomorphisms requires the anomaly to be a closed one form on $\operatorname{Diff}_{0}(m)$. Put another way, the one form $\eta=$ $d\left(\operatorname{det} B_{\phi \cdot g}\right) /\left(\operatorname{det} B_{\phi \cdot g}\right)$ is closed and represents an element in the first cohomology class of $\operatorname{Diff}_{0}(M)$.

In reference [8] we show that there is a relation between $\eta$ and $c_{1}\left(\operatorname{Ind} \mathbf{B}_{\rho}\right)$. The connection is obtained by using the transgression operation [11]. We give a brief 
description. The first Chern class of the index bundle is represented by a closed two form on $\mathfrak{M} / \operatorname{Diff}_{0}(M)$ when computed in terms of curvature. By using the transgression operation one obtains a closed one form on Diff ${ }_{0}(M)$. Explicit formulas may be found in $[6,12-14]$. The transgression of $c_{1}$ and $\eta$ give the same cohomology class in the sense of local cohomology (see below). To check whether the gravitational anomalies cancel, we need check only that the first Chern classes of the corresponding Ind $\mathbf{B}_{\rho}$ cancel. We use the right-hand side of (2.4) to calculate the first Chern class of the index bundle.

Local cohomology is relevant here because the first cohomology of $\operatorname{Diff}_{0}\left(S^{4 k-2}\right)$ is finite, i.e., has only torsion for $k=1$ and $k \geqslant 6$. As far as we can tell, no complete proof is in the literature, see $[15,16]$. One expects the result to be true for all $k$. When the first cohomology is finite, the one-form $\eta$ is exact, i.e., $\log \operatorname{det} B_{\phi \cdot g}$ is a well defined single valued function of $\phi$. For a topologist, there is no anomaly. The important physical question is whether one can write the anomaly as the differential of a local function of $\phi$. We have shown [8] that this is not possible by using a generalization of Gilkey's lemma $[17,18]$. Hence $\eta$ represents nontrivial class in the localone-cohomology of $\operatorname{Diff}_{0}(M)$. The notion of locality is familiar to physicists since it is intimately related to anomalies and to the renormalization program for gauge theories [19-21]. Local cohomology has been defined in reference [22].

Consider the combined Lorentz and Einstein anomalies. Mathematically this is the situation where one allows all automorphisms $\mathscr{H}$ of a principal bundle leaving a point fixed. The group of gauge transformations $\mathscr{G}$ is a subgroup and the quotient group $\mathscr{H} / \mathscr{G}$ contains Diff 0 (M). For the special case of the bundle of bases $B$ of $M$, $\operatorname{Diff}_{0}(M)$ can be lifted and $\mathscr{H}$ is a semi-direct product of $\mathscr{G}$ and $\operatorname{Diff}_{0}(M)$. To use the orthogonal group instead of the linear group one has to consider the subset of $B \times \mathfrak{M}$ consisting of orthogonal frames in the metric $g$.

In this case, the Dirac operator depends on a metric and a compatible connection. We dwell on this extension for the following reason. Now the anomaly is a closed one form on $\mathscr{G} \times \operatorname{Diff}_{0}(M)$. Its restriction to $\mathscr{G}$ is invariant under $\operatorname{Diff}_{0}(M)$ and is the usual Lorentz anomaly. It is the anomaly for a $\mathrm{SO}(4 k-2)$ gauge theory. However, one can add a local counterterm to the vacuum functional so that the new vacuum functional is invariant under $\mathscr{G}$ but not under $\operatorname{Diff}_{0}(M)$. In this way one gets the Einstein anomaly. The transfer process is reversible [14]. It appears one can represent the local cohomology of the semi-direct product as an Einstein anomaly or as a Lorentz anomaly. The manifestation of the anomaly on $\mathscr{G} \times \operatorname{Diff}_{0}(M)$ as a Lorentz anomaly has a topological interpretation. The relationships between the topological and the local interpretation of anomalies will be discussed in [8].

\section{Calculations}

We have to choose the vector bundle $V$ in such a way that the domain of the operator $B_{g}$ is either chiral spin $1 / 2$ fermions, chiral spin $3 / 2$ fermions, or the self dual antisymmetric tensor field. We discuss the different cases individually. For future convenience we also discuss the Euler complex and the Hirzebruch complex.

Common to all the cases is the following observation. The structure group of the spin frame bundle of $Z$ is $\operatorname{Spin}(2 n+2)$. Since $Z$ is a fiber bundle, the structure group 
may be reduced to $\operatorname{Spin}(2 n) \times \operatorname{Spin}(2)$. This means that the values of the curvature two form takes the following form:

$$
\left(\begin{array}{cc}
\mathfrak{s p i n}(2 n) & 0 \\
0 & \mathfrak{s p i n}(2)
\end{array}\right)
$$

As a consequence the $\hat{A}$ genus may be factored as

$$
\hat{A}(Z)=\frac{y / 2}{\sinh (y / 2)} \prod_{j=1}^{n} \frac{x_{j} / 2}{\sinh \left(x_{j} / 2\right)},
$$

where $y$ is the Spin (2) piece and $\left\{x_{j}\right\}$ are the $\operatorname{Spin}(2 n)$ pieces. Note that if heuristically one thinks of each $x_{j}$ and each $y$ as being a two form, then $x$ and $y$ are not differential forms on $M$ and $Y$ respectively. They contain mixed pieces.

\section{Case I. Dirac Complex}

$$
\operatorname{ch}(\text { Ind }[\text { Dirac }])=\int_{M} \frac{y / 2}{\sinh (y / 2)} \prod_{j=1}^{n} \frac{x_{j} / 2}{\sinh \left(x_{j} / 2\right)} .
$$

The $\hat{A}$ genus is determined by the Pontrjagin classes and therefore it defines forms of degrees which are divisible by four. For our purposes this will be important since $Z$ is a manifold of dimension $4 k$. Similar remarks apply to the remaining cases.

\section{Case II. Hirzeburch Complex}

The complexification of the self dual differential forms on $M$ may be identified with $S_{M}^{+} \otimes\left(S_{M}^{+} \oplus S_{M}^{-}\right)$. The anti-dual forms are given by $S_{M}^{-} \otimes\left(S_{M}^{+} \oplus S_{M}^{-}\right)$. The Hirzebruch signature operator may be identified as

$$
B_{g}: C^{\infty}\left(S_{M}^{+} \otimes\left(S_{M}^{+} \oplus S_{M}^{-}\right)\right) \rightarrow C^{\infty}\left(S_{M}^{-} \otimes\left(S_{M}^{+} \oplus S_{M}^{-}\right)\right) .
$$

It follows that the vector bundle $V$ is given by $V=S_{M}^{+} \oplus S_{M}^{-}$. The Chern character of $V$ is given by Eq. (5.1) of [23]:

$$
\operatorname{ch}\left(S_{M}^{+} \oplus S_{M}^{-}\right)=\prod_{j=1}^{n} 2 \cosh \left(x_{j} / 2\right) .
$$

It is important to notice that since $V$ is a bundle over $M$ only the $x$ 's enter in (4.4). The family's index theorem gives

$$
\operatorname{ch}(\operatorname{Ind}[\text { Hirz }])=\int_{M} \frac{y / 2}{\sinh (y / 2)} \prod_{j=1}^{n} \frac{x_{j}}{\tanh \left(x_{j} / 2\right)} .
$$

Naturally the above formula involves Hirzeburch's $L$-polynomial $[9,23]$.

\section{Case III. Euler Complex}

The Euler complex is defined by mapping even forms to odd forms using the differential operator $d+d^{*}$. The complexification of even forms on $M$ may be identified with $\left(S_{M}^{+} \otimes S_{M}^{+}\right) \oplus\left(S_{M}^{-} \otimes S_{M}^{-}\right)$, and the complexification of the odd forms 
with $\left(S_{M}^{-} \otimes S_{M}^{+}\right) \oplus\left(S_{M}^{+} \otimes S_{M}^{-}\right)$. As far as the index theorem is concerned, the operator

$$
B_{g}: C^{\infty}\left(\left(S_{M}^{+} \otimes S_{M}^{+}\right) \oplus\left(S_{M}^{-} \otimes S_{M}^{-}\right)\right) \rightarrow C^{\infty}\left(\left(S_{M}^{-} \otimes S_{M}^{+}\right) \oplus\left(S_{M}^{+} \otimes S_{M}^{-}\right)\right)
$$

may be viewed as a map

$$
B_{g}: C^{\infty}\left(S_{M}^{+} \otimes\left(S_{M}^{+}-S_{M}^{-}\right)\right) \rightarrow C^{\infty}\left(S_{M}^{-} \otimes\left(S_{M}^{+}-S_{M}^{-}\right)\right) .
$$

Equation (4.7) is obtained from (4.6) by moving terms from one side of the arrow to the other. The vector bundle $V$ now becomes the virtual bundle or formal difference $S_{M}^{+}-S_{M}^{-}$. According to Eq. (5.1) of [23] one has

$$
\operatorname{ch}\left(S_{M}^{+}-S_{M}^{-}\right)=\prod_{j=1}^{n} 2 \sinh \left(x_{j} / 2\right) \text {. }
$$

The family's index theorem gives

$$
\operatorname{ch}(\operatorname{Ind}[\text { Euler }])=\int_{M} \frac{y / 2}{\sinh (y / 2)} \prod_{j=1}^{n} x_{j} .
$$

One can immediately see that $c_{1}$ is zero in the case of interest. The Euler form

$$
e=\prod_{j=1}^{n} x_{j}
$$

is a $2 n$-form, the term involving the $y$ is formally a linear combination of forms of degree $0,4,8, \ldots$. Therefore after integration over $M$ one cannot be left with a two form. In fact we have just proven a special case of a more general theorem: the characteristic classes for the index bundle of the Euler complex all vanish. The reason is that Euler is topological in nature and varying the metric should have no effect.

\section{Case IV. The Self-Dual Complex}

In the supergravity theories [24] one encounters self-dual tensors. It is important to realize that these theories are defined on a manifold of dimension $4 k-2$ with a Lorentz metric. The self-dual tensor is a $2 k-1$ form.

To apply the family's index theorem we analytically continue naively to a metric of Euclidean signature. The complexification of the anti-dual even forms is $S_{M}^{-} \otimes S_{M}^{-}$, while the self-dual odd forms are given by $S_{M}^{+} \otimes S_{M}^{-}$. We are interested in

$$
B_{g}: C^{\infty}\left(S_{M}^{+} \otimes S_{M}^{-}\right) \rightarrow C^{\infty}\left(S_{M}^{-} \otimes S_{M}^{-}\right) .
$$

The vector bundle $V$ is given by $S_{M}^{-}$. One has the following formal manipulations which are valid in $K$-theory:

$$
\begin{aligned}
\operatorname{ch}\left(S_{M}^{-}\right) & =(1 / 2) \operatorname{ch}\left(\left(S_{M}^{+} \oplus S_{M}^{-}\right)-\left(S_{M}^{+}-S_{M}^{-}\right)\right) \\
& =(1 / 2) \operatorname{ch}\left(S_{M}^{+} \oplus S_{M}^{-}\right)-(1 / 2) \operatorname{ch}\left(S_{M}^{+}-S_{M}^{-}\right) \\
& =(1 / 2) \prod_{j=1}^{n} 2 \cosh \left(x_{j} / 2\right)-(1 / 2) \prod_{j=1}^{n} 2 \sinh \left(x_{j} / 2\right)
\end{aligned}
$$

by (4.4) and (4.8). Comparing to Cases II and III we see that the self dual complex 
may be thought as being given by one half of Hirzebruch minus one half of Euler. We immediately have the result

$$
\operatorname{ch}(\operatorname{Ind}[\operatorname{self} \mathrm{dual}])=\frac{1}{2} \int_{M} \frac{y / 2}{\sinh (y / 2)} \prod_{j=1}^{n} \frac{x_{j}}{\tanh \left(x_{j} / 2\right)},
$$

since the Euler contribution vanishes.

\section{Case V. Rarita-Schwinger Complex}

Naively the Rarita-Schwinger complex is given by

$$
B_{g}: C^{\infty}\left(S_{M}^{+} \otimes T^{*} M\right) \rightarrow C^{\infty}\left(S_{M}^{-} \otimes T^{*} M\right) .
$$

However, $S_{M}^{+} \otimes T^{*} M$ is not pure spin $\frac{3}{2}$. One must remove two spin $\frac{1}{2}$ degrees of freedom of the same chirality and one spin $\frac{1}{2}$ degree of freedom of the opposite chirality [25]. The relevant elliptic complex becomes

$$
\begin{aligned}
B_{g}: C^{\infty}\left(S_{M}^{+} \otimes T^{*} M\right)-C^{\infty}\left(S_{M}^{+}\right)-C^{\infty}\left(S_{M}^{+}\right)-C^{\infty}\left(S_{M}^{-}\right) \\
\quad \rightarrow C^{\infty}\left(S_{M}^{-} \otimes T^{*} M\right)-C^{\infty}\left(S_{M}^{-}\right)-C^{\infty}\left(M_{M}^{-}\right)-C^{\infty}\left(S_{M}^{+}\right) .
\end{aligned}
$$

By manipulating terms just as we did in the Euler case one finds

$$
B_{g}: C^{\infty}\left(S_{M}^{+} \otimes\left(T^{*} M-1\right)\right) \rightarrow C^{\infty}\left(S_{M}^{-} \otimes\left(T^{*} M-1\right)\right) .
$$

The vector bundle $V$ is identified with the formal difference $T^{*} M-1$ and it follows that

$$
\operatorname{ch}\left(T^{*} M-1\right)=\operatorname{ch}\left(T^{*} M\right)-1=2 \sum_{j=1}^{n} \cosh \left(x_{j}\right)-1 .
$$

The family's index theorem becomes

$$
\begin{aligned}
\operatorname{ch}(\text { Ind [Rarita-Schwinger }])= & \int_{M} \frac{y / 2}{\sinh (y / 2)}\left(\prod_{j=1}^{n} \frac{x_{j} / 2}{\sinh \left(x_{j} / 2\right)}\right) \\
& \cdot\left(2 \sum_{i=1}^{n} \cosh \left(x_{i}\right)-1\right) .
\end{aligned}
$$

\section{The Results of Alvarez-Gaumé and Witten}

In Sect. III we reported that the anomaly was related to the first Chern class of the index bundle via the transgression operation. If we can arrange the field content in such a way that the total contribution to the Chern class is zero then we have an anomaly free theory. This requires that we carefully collate the results of Sect. IV, making sure that we have all the relative signs correctly and a certain factor of two. All we are really concerned with are the $x$-dependent terms in the formulas of Sect. IV since the $y$ term is common to all formulas. We will collate the $x$-dependent terms of all the integrands. We have to interpret the formulas of Sect. IV as living in a manifold of Lorentz signature.

The contribution to the anomaly due to a spin $\frac{1}{2}$ negative chirality fermion is 
given by

$$
I_{1 / 2}=(-1)^{2} \prod_{j=1}^{n} \frac{x_{j} / 2}{\sinh \left(x_{j} / 2\right)} .
$$

One minus sign is just the Fermi $(-1)$ relative to the bosonic contribution. The second one is due to the fact that the calculation in Sect. IV was done for positive chirality. Likewise, the contribution due to a negative chirality spin $\frac{3}{2}$ fermion is given by

$$
I_{3 / 2}=(-1)^{2}\left(\prod_{j=1}^{n} \frac{x_{j} / 2}{\sinh \left(x_{j} / 2\right)}\right)\left(2 \sum_{i=1}^{n} \cosh \left(x_{i} / 2\right)-1\right) .
$$

The only tricky calculation is the anti-symmetric self dual tensor field $F$. One should think of $F$ as coming from a potential $A, F=d A$. The potential is an even form, and therefore (4.11) was a map in the opposite direction. This means we should take Eq. (4.13) with a minus sign:

$$
I_{A}=(-1) \frac{1}{2} \frac{1}{2} \prod_{j=1}^{n} \frac{x_{j}}{\tanh \left(x_{j} / 2\right)}
$$

There is no Fermi $(-1)$ since the particles are bosons. There is an additional factor of $\left(\frac{1}{2}\right)$ since a self dual tensor in a $4 k-2$ dimensional Lorentz manifold may be taken to be real. The calculations in Sect. IV were performed in the complexified deRham complex. This factor may be understood by counting the number of physical degrees of freedom or by analyzing Feynman diagrams [1]. To make our results agree with Eq. (114) of reference [1] one notices that the term of order $2 k$ in $x$ of (5.3) is the same as the term of order $2 k$ in the quantity (note that $2 n=4 k-2$ ):

$$
-\frac{1}{8} \prod_{j=1}^{n} \frac{x_{j}}{\tanh \left(x_{j}\right)}
$$

The reason why one is interested in the term of order $2 k$ in $x$ is that one needs a $4 k$ form to integrate over the $4 k-2$ dimensional manifold $M$ to obtain a two form on $Y$. The order $2 k$ term is all one needs to obtain the leading term of the first characteristic class, i.e., the approximation that

$$
\frac{y / 2}{\sinh (y / 2)}=1+O\left(y^{2}\right)
$$

The rest of the terms may be obtained by using the consistency condition $[6,10,14]$.

Except for an overall factor which does not affect the striking cancellation in the ten dimensional chiral $N=2$ supergravity theory, the above are the main results of reference [1]. Our computation gives an extra factor of $\frac{1}{2}$ in the Alvarez-Gaumé and Witten formulas when the latter are interpreted as the covariant form [14] of the anomaly. If one tries to interpret their formulas as the linearized consistent form [14] of the anomaly, then we find a further dimension dependent factor of $1 /(2 k)$.

Acknowledgements. We would like to thank W. Hsiang, K. Igusa and R. Lashof for discussions on the topology of the diffeomorphism group, and R. Stora for helpful comments. O.A. would like to thank D. Freed for useful conversations and the A.P. Sloan Foundation for support. 


\section{References}

1. Alvarez-Gaumé, L., Witten, E.: Gravitational anomalies. Nucl. Phys. B 234, 269 (1983)

2. Atiyah, M. F., Singer, I. M.: Ann. Math. 93, 119 (1971)

3. Atiyah, M. F., Singer, I. M.: Dirac operators to vector potentials. Proc. Natl. Acad. Sci. 81, 2597 (1984)

4. Alvarez-Gaumé, L., Ginsparg, P.: The topological meaning of non-Abelian anomalies. Harvard Preprint HUTP-83/A018 (1983)

5. Lott, J.: Harvard Mathematics Preprint (1984)

6. Stora, R.: Cargèse Lectures (1983)

7. Hitchin, N.: Adv. Math. 14, 1 (1974)

8. Alvarez, O., Singer, I. M., Zumino, B.: (in preparation)

9. Eguchi, T., Gilkey, P. B., Hanson, A. J.: Gravitation, gauge theories differential geometry. Phys. Rep. 66, 213 (1980)

10. Wess, J., Zumino, B.: Consequences of anomalous Ward identities. Phys. Lett. 37B, 95 (1971)

11. Chern, S.-S.: Complex manifolds without potential theory. New York: Springer 1979

12. Zumino, B., Yong-Shi, Wu, Zee, A.: Univ. of Washington Preprint (1983), Nucl. Phys. B to be published.

13. Zumino, B.: Les Houches lectures (1983) Stora, R., De Witt, B. (eds.). North-Holland (to be published)

14. Bardeen, W. A., Zumino, B.: LBL Preprint LBL-17639 (1984)

15. Dennis, R. K., Igusa, K.: Algebraic K-theory, Proceedings of the Oberwolfach Conference, Vol. 966. New York: 1982

16. Hsiang, W.: Geometric topology. New York: Academic Press 1979

17. Gilkey, P. B.: Adv. Math. 10, 344 (1973)

18. Atiyah, M. F., Bott, R., Patodi, V. K.: Invent. Math. 19, 279 (1973)

19. Bardeen, W. A.: Anomalous Ward identities in spinor field theories. Phys. Rev. 184, 1848 (1969)

20. Gross, D. J., Jackiw, R.: Effect of anomalies on quasi-renormalizale theories. Phys. Rev. D6, 477 (1972)

21. Bouchiat, C., Iliopoulos, J., Meyer, Ph.: An anomaly-free version of Weinberg's model. Phys. Lett. 38B, 519 (1972)

22. Bonora, L., Cotta-Ramusino, P.: Some remarks on BRS transformations, anomalies and the cohomology of the Lie algebra of the group of gauge transformations. Commun. Math. Phys. 87, 589 (1983)

23. Atiyah, M. F., Singer, I. M.: Ann. Math. 87546 (1968)

24. Green, M. B., Schwarz, J. H.: Supersymmetrical dual string theory. Nucl. Phys. B181, 502 (1981); Supersymmetrical dual string theory (II). Vertices and trees. B198, 252 (1982); and-_- (III). Loops and renormalization, B198, 441 (1982); Supersymmetrical string theories. Phys. Lett. 109B, 444 (1982)

25. Römer, H.: Axial anomaly and boundary terms for general spinor fields. Phys. Lett. 82B, 172 (1979)

Communicated by R. Stora

Received May 14, 1984 
